\title{
Anomalous planar Hall effect in two-dimensional trigonal crystals
}

\author{
Raffaele Battilomo, ${ }^{1}$ Niccoló Scopigno $\odot,{ }^{1}$ and Carmine Ortix $\circledast^{1,2}$ \\ ${ }^{1}$ Institute for Theoretical Physics, Center for Extreme Matter and Emergent Phenomena, Utrecht University, Princetonplein 5 , \\ 3584 CC Utrecht, The Netherlands \\ ${ }^{2}$ Dipartimento di Fisica “E. R. Caianiello,” Universitá di Salerno, IT-84084 Fisciano, Italy
}

(Received 30 April 2020; revised 1 August 2020; accepted 23 December 2020; published 19 January 2021)

\begin{abstract}
The planar Hall effect (PHE) is the appearance of an in-plane transverse voltage in the presence of coplanar electric and magnetic fields. Its hallmark is a characteristic $\pi$ periodic, i.e., even under a magnetic-field reversal, angular dependence with the transverse voltage that exactly vanishes when the electric and magnetic fields are aligned. Here we demonstrate that in two-dimensional trigonal crystals Zeeman-induced nontrivial Berry curvature effects yield a previously unknown anomalous PHE that is odd in the magnetic field and independent of the relative angle with the driving electric field. We further show that when an additional mirror symmetry forces the transverse voltage to vanish in the linear-response regime, the anomalous PHE can occur as a second-order response at both zero and twice the frequency of the applied electric field. We demonstrate that this nonlinear PHE possesses an antisymmetric quantum contribution that originates from a Zeeman-induced Berry curvature dipole.
\end{abstract}

DOI: 10.1103/PhysRevResearch.3.L012006

Introduction. The Hall effect arises when the conduction electrons of a solid acquire a transverse velocity either due to an externally applied magnetic field or an intrinsic ordered magnetic structure. The associated Hall conductivity is encoded in the antisymmetric dissipationless part of the conductivity tensor, which, for a two-dimensional (2D) system, is given by the single scalar $\sigma_{\mathrm{H}}=\left(\sigma_{x y}-\sigma_{y x}\right) / 2$. Onsager reciprocity relations force $\sigma_{\mathrm{H}}$ to vanish in time-reversal symmetric conditions. In addition $\sigma_{\mathrm{H}}$ transforms as a pseudoscalar under a generic spatial point-group symmetry operation. Hence, to observe a Hall response it is necessary to break beside the time-reversal invariance all mirror symmetries. These conditions are immediately met in the ordinary classical Hall effect where an out-of-plane magnetic field is applied. In this configuration a net Lorentz force grants the electron a transverse velocity and, consequently, a finite Hall voltage. On the other hand, a magnetic field coplanar with the driving electric field cannot generate a Lorentz force bending the electron trajectories. Nevertheless, transverse currents can and do still exist in strongly spin-orbit coupled systems displaying a sizable anisotropy in the magnetoconductance. This magnetotransport phenomenon, known as planar Hall effect (PHE), does not contribute to the dissipationless Hall conductivity $\sigma_{\mathrm{H}}$ but manifests itself in the symmetric contribution of the conductivity tensor: It cannot be qualified as a genuine Hall effect. In the majority of (quasi)-two-dimensional systems, the PHE has an entirely semiclassical origin and has been shown to

Published by the American Physical Society under the terms of the Creative Commons Attribution 4.0 International license. Further distribution of this work must maintain attribution to the author(s) and the published article's title, journal citation, and DOI. arise in thin films of ferromagnetic semiconductors [1-3] and two-dimensional electron gases formed at perovskite oxide interfaces [4,5]. Band anisotropies have also been proposed as the source of the PHE in thin films of antiferromagnetic semiconductors [6]. Moreover the PHE plays a central role in the transport properties [7-9] of Weyl semimetals [10-19]. In these topological semimetals, the induced transverse Hall voltage, the applied current, and the magnetic field all lie on the same plane, precisely in a configuration in which the conventional Hall effect vanishes. Even more importantly, the PHE in Weyl semimetals is a prime physical consequence of the chiral anomaly of Weyl fermions [20-25]. The conducting surfaces of three-dimensional topological insulators (3DTIs) [26] have also been recently shown to support a PHE $[27,28]$. In these materials, an external planar magnetic field conspires with the spin-momentum locking of the Dirac cones to produce a strongly directional-dependent net transverse current. All these studies established a paradigm for the planar Hall effect: (i) a $\sin 2 \theta$ angular dependence with $\theta$ representing the relative angle between the applied electric and the magnetic fields and (ii) a magnitude set precisely by the anisotropy in the longitudinal magnetoresistance. However, a PHE beyond this paradigm is, in principle, symmetry allowed. Beside timereversal invariance, a planar magnetic field can potentially break all mirror symmetries present in the solid-state structure. Therefore, a planar magnetic field is entitled to generate a dissipationless Hall conductance.

In this Letter, we demonstrate that 2D materials with strong spin-orbit coupling and crystalline trigonal symmetry possess a previously overlooked anomalous planar Hall effect (APHE). This effect unique to trigonal crystals, derives directly from the "bending" of the electron trajectories encoded in the geometric properties of the electronic wave functions [29] - the APHE stems from a Zeeman-induced nontrivial 


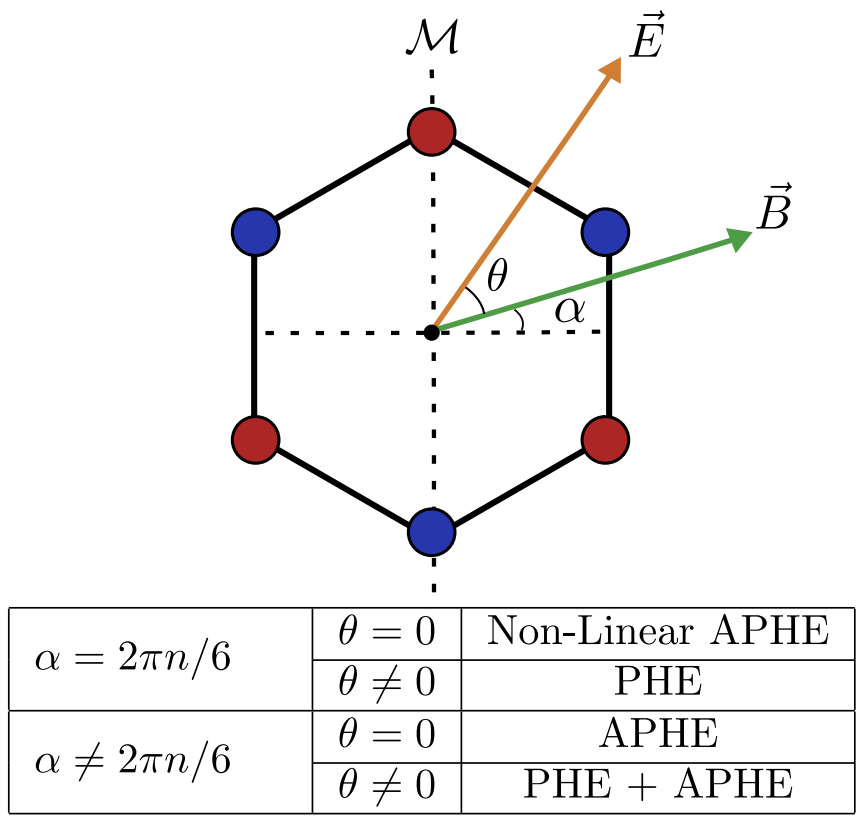

FIG. 1. Schematic of the conventional and anomalous PHEs in a two-dimensional trigonal crystal as a function of the relative angle $\theta$ between the electric $(\vec{E})$ and the magnetic $(\vec{B})$ fields and the crystallographic angle $\alpha$ determined by $\vec{B}$ and the mirror line $\mathcal{M}$.

Berry curvature profile. Besides possessing the antisymmetric properties of conventional Hall conductivities, i.e., $\sigma_{x y} \rho_{y x}=$ -1 , we show that the APHE is independent of the relative direction between the driving electric field and the in-plane magnetic field. Therefore, as shown in Fig. 1, these anomalous planar Hall currents persist even when the two fields are collinear and the conventional planar Hall currents vanish. We also show that when mirror symmetries constrain the APHE to vanish (Fig. 1), transverse Hall currents are still present: They arise in the nonlinear response regime and manifest as a second-harmonic response to an oscillating electric field. In strict analogy with the nonlinear Hall effect of time-reversal invariant materials [30-40], we find that this nonlinear APHE has a geometric contribution that is directly related to the first moment of the Berry curvature, the so-called Berry curvature dipole [31]. This clearly distinguishes the nonlinear response we discuss here with the one recently shown to exist on the surface of 3DTIs [28].

We propose graphene and transition-metal dichalcogenides (TMDs) monolayers [41-43] where transition-metal and chalcogen atoms form trigonal crystal structures as possible material platforms that can host both the linear and the nonlinear APHEs in the presence of Rashba spin-orbit coupling [44]. In these systems, the absence of inversion symmetry results in massive Dirac cones with a sizable Berry curvature. We find that the Berry curvature-induced APHE vanishes only when the magnetic field is perpendicular to a mirror line of the trigonal crystal. In this situation, however, the finite Berry curvature dipole being still finite provides a nonlinear anomalous PHE.

Anomalous planar Hall effect. Within the quasiclassical Boltzmann picture of transport, the transverse conductivity $\sigma_{y x}$ in the presence of coplanar electric and magnetic fields can be written as $[8,45]$

$$
\begin{aligned}
\sigma_{y x}= & e^{2} \int \frac{d^{3} k}{(2 \pi)^{3}} D \tau\left(-\frac{\partial f_{e q}}{\partial \epsilon}\right)\left\{\left[v_{y}+\frac{e B \sin \theta}{\hbar}\left(\mathbf{v}_{\mathbf{k}} \cdot \boldsymbol{\Omega}_{\mathbf{k}}\right)\right]\right. \\
& \left.\times\left[v_{x}+\frac{e B \cos \theta}{\hbar}\left(\mathbf{v}_{\mathbf{k}} \cdot \boldsymbol{\Omega}_{\mathbf{k}}\right)\right]\right\}+\frac{e^{2}}{h} \int \frac{d^{3} k}{\left(2 \pi^{3}\right)} \Omega_{k}^{z} f_{e q},(1)
\end{aligned}
$$

where $f_{e q}$ is the equilibrium Fermi-Dirac distribution. In the equation above one can distinguish three different contributions: There is a first purely semiclassical term given by the weighted integral of the electronic velocities $v_{x} v_{y}$ that remains finite for strongly spin-orbit coupled and Zeeman spin-split electronic bands. This term is responsible for the PHE observed in (anti)ferromagnetic semiconductors and at oxide interfaces. The remaining contributions come about due to the anomalous velocity of Bloch electrons [46] and are, therefore, directly related to the Berry curvature $\boldsymbol{\Omega}(\mathbf{k})$. Specifically the terms containing the product $\boldsymbol{\Omega}(\mathbf{k}) \cdot \mathbf{v}(\mathbf{k})$ are responsible for the PHE in three-dimensional topological semimetals. Precisely as the classical contribution, the Berry curvatureinduced PHE in Dirac and Weyl semimetals is even in the applied planar magnetic field, i.e., $\sigma_{x y}(B) \equiv \sigma_{x y}(-B)$ and, hence, does not satisfy the antisymmetry property of the conventional Hall conductivity. Finally, the last term given by the integral of the Berry curvature over the Fermi surface of the occupied states corresponds to the anomalous Hall effect characteristic of time-reversal broken materials that can be singled out by taking measurements at $B \equiv 0$. In two-dimensional materials since $\mathbf{v}_{\mathbf{k}} \perp \boldsymbol{\Omega}(\mathbf{k})$, the PHE is conventionally assumed to not possess any Berry curvature-induced contribution. Put differently the PHE of two-dimensional systems should not represent a topological response function. However, as we will show below, this conventional wisdom has to be reevaluated in two-dimensional materials with a trigonal symmetry. The crux of the story is that the Zeeman spin splitting of the electronic bands induced by the planar magnetic field triggers a nonvanishing Berry curvature and, thus, engenders a planar Hall voltage that is entirely of quantum origin. In the linearresponse regime the consequence of this is twofold. First, this transverse conductance does obey the antisymmetry property of the conventional Hall conductance. Second, the transverse voltage is completely independent of the relative direction between the two coplanar fields. We dub this topological response the anomalous planar Hall effect: It can be distinguished by the anomalous Hall effect by taking measurements at both $B \neq 0$ and $B \equiv 0$, and it can be singled out from the conventional PHE of two-dimensional systems by aligning the external magnetic and electric fields, or taking measurements at both $+B$ and $-B$.

As the Zeeman-induced Berry curvature obeys the symmetry properties of the crystal, point-group symmetries can force the APHE response to vanish. Consider, for instance, a two-dimensional system subject to a planar magnetic field perpendicular to a mirror line of the crystal $(\alpha=0$ in Fig. 1), which, without loss of generality, we assume to map a point with coordinates $\{x, y\}$ to $\{-x, y\}$. Since the external planar magnetic field preserves the mirror symmetry $\mathcal{M}_{x}$, the Berry curvature will obey the symmetry constraint $\Omega_{z}\left(k_{x}, k_{y}\right)=-\Omega_{z}\left(-k_{x}, k_{y}\right)$ even when the Zeeman 
spin splitting of the bands is fully taken into account. Furthermore, the Fermi surface must be symmetric with respect to the mirror line, and, therefore, the integral of the Berry curvature is forced to vanish. This, however, does not automatically imply the absence of a transverse planar Hall current when the driving electric field and the external magnetic field are collinear. The existence of a single residual mirror line still allows for a finite Berry curvature dipole defined by

$$
D_{b d}=\int_{k} f_{0}\left(\partial_{b} \Omega_{d}\right),
$$

which will be directed perpendicular to the residual mirror line and, thus, aligned with the magnetic and electric fields. In analogy with the quantum nonlinear Hall effect in time-reversal symmetric conditions [31], a finite Berry curvature dipole causes a two-dimensional crystal subject to an $\mathrm{AC}$ driving electric field $E_{c}=\operatorname{Re}\left(\mathcal{E}_{c} e^{i \omega t}\right)$ to develop an additional nonlinear current $j_{a}=\operatorname{Re}\left(j_{a}^{0}+j_{a}^{2 \omega} e^{2 i \omega t}\right)$ characterized by two Fourier components at zero and twice the frequency of the applied external field: $j_{a}^{0}=\chi_{a b c} \mathcal{E}_{b} \mathcal{E}_{c}^{*}$ and $j_{a}^{2 \omega}=\chi_{a b c} \mathcal{E}_{b} \mathcal{E}_{c}$. The response function $\chi_{a b c}$ has a quantum origin in the Berry curvature dipole and can be expressed as $\chi_{a b c}=-\epsilon_{a d c} e^{3} \tau D_{b d} / 2(1+i \omega \tau), \epsilon_{a d c}$ being the Levi-Civita tensor and $\tau$ the scattering time. This quantum nonlinear APHE coexists with a semiclassical second-order but Berryphase independent contribution to the transverse nonlinear Hall conductivity [45]. The latter can be distinguished from the former since the Berry curvature dipole contributes to the antisymmetric dissipationless part of the nonlinear Hall conductivity vector, defined as $\chi_{c}=\epsilon_{a b} \chi_{a b c} / 2$, whereas the semiclassical contribution is contained in the symmetric part of the response [47]. Finally, we emphasize that producing a nonvanishing dipole does not require a crystalline symmetry content as low as the one required in time-reversal symmetric conditions. This is because the externally applied planar magnetic field breaks all rotational and additional mirror symmetries, thus, partially relaxing the necessary conditions for a finite dipole. As a result, the nonlinear Hall currents generated by the Berry curvature dipole vanish when the external magnetic field is set to zero, thus, showing that this effect is a genuine Hall one.

Symmetry analysis. We now show that the (non)linear APHE naturally arises in strongly spin-orbit coupled 2D crystals with $\mathcal{C}_{3 v}$ symmetry. First, we note that a planar magnetic field is invariant under the combined $\mathcal{C}_{2} \mathcal{T}$ symmetry, where $\mathcal{C}_{2}$ indicates the twofold rotation around the axis perpendicular to the crystalline plane and $\mathcal{T}$ is the internal time-reversal symmetry. The presence of $\mathcal{C}_{2} \mathcal{T}$ symmetry then forces the Berry curvature to be identically zero: $\Omega_{z}(k) \equiv 0$. As a result, only trigonal crystals, which do not contain a twofold rotation symmetry, can display a planar magnetic-field induced nontrivial Berry curvature. Another necessary condition for the appearance of a finite Berry curvature dipole is the presence of a sizable spin-orbit coupling, which ensures that the crystal Hamiltonian $\mathcal{H}_{0}$ and the Zeeman coupling term $\mathcal{H}_{Z}=\vec{B} \cdot \vec{\sigma}$ do not commute. This prevents the possibility of separating the Bloch eigenfunctions of the full Hamiltonian $\mathcal{H}=\mathcal{H}_{0}+$ $\mathcal{H}_{Z}$ into a spinorial part $\chi_{s}$, regulated only by the Zeeman term, and an orbital wave-function $\psi_{\text {orb }}\left(k_{x}, k_{y}\right)$ where all the momentum dependence is stored: For eigenstates of that form the Berry curvature is indeed independent from the Zeeman coupling and retains the trigonal symmetry of the pristine crystal also in the presence of the externally applied magnetic field. This forces the corresponding Berry curvature dipole to vanish. Finally, we note that the nonlinear PHE can occur only if the $\mathcal{S U}(2)$ spin symmetry in $\mathcal{H}_{0}$ is completely broken. A residual $\mathcal{U}(1)$ spin symmetry_as ensured by a mirror plane symmetry $\mathcal{M}_{z}-$ would, in fact, imply that $\mathcal{H}_{0}$ commutes with the spin rotation $\mathcal{U}_{\alpha}=e^{i \alpha \sigma_{z} / 2}$. This operator rotates the planar magnetic field by an angle $\alpha$ according to $\vec{B}^{\prime}=\mathcal{R}_{\alpha}(\vec{B})$, but since $\mathcal{U}_{\alpha}$ does not explicitly contain a momentum dependence, the two Hamiltonians $\mathcal{H}(\vec{B})$ and $\mathcal{H}^{\prime}=\mathcal{U}_{\alpha}^{\dagger} \mathcal{H}(\vec{B}) \mathcal{U}_{\alpha} \equiv \mathcal{H}\left(\vec{B}^{\prime}\right)$ have the same Berry curvature dipole. On the other hand, the dipole is forced to be parallel to the external magnetic field when the latter is orthogonal to a mirror line [48]. If we choose $\vec{B}$ and $\vec{B}^{\prime}$ to be perpendicular to different mirror lines (any two among the three of the $\mathcal{C}_{3 v}$ crystal), the only allowed vector compatible with such a constraint is the null one. Hence, the Berry curvature dipole must vanish thereby proving that the nonlinear planar Hall effect necessitates a complete breaking of the spin-rotation symmetry.

Having established the occurrence of a quantum nonlinear PHE when the system is characterized by a residual mirror symmetry, we now consider the situation in which the external planar magnetic field is not constrained to be orthogonal to one of the three mirror lines of the $\mathcal{C}_{3 v}$ crystal. Since the presence of the planar magnetic field reduces the point group to the trivial group $\mathcal{C}_{1}$, the Berry curvature does not obey any constraint, and, therefore, the net anomalous velocity is not forced to vanish. This, consequently, leads to the possibility of a purely Zeeman-induced quantum PHE in the linear-response regime, which represents an antisymmetric contribution to the resistivity tensor and, therefore, displays a $2 \pi$-periodic angular dependence. Furthermore, it is important to note that for the integral of the Berry curvature weighed by the equilibrium Fermi distribution function to be nonzero, the spin-rotation symmetry needs to be completely broken-in a crystal with a $\mathcal{M}_{z}$ mirror plane, the combined $\mathcal{M}_{z} \mathcal{T}$ symmetry, which is still preserved with a planar magnetic field, forces the Berry curvature to be an odd function. Hence, as for its nonlinear counterpart, also the quantum PHE in linear response can only occur in strongly spin-orbit coupled crystals. It is, thus, expected to coexist with the conventional Berry-phase independent contribution to the PHE, which, as stated above, represents instead a symmetric part of the resistivity tensor. These different symmetry properties of the quantum and semiclassical contributions to the linear PHE imply that the semiclassical linear contribution to the PHE can be isolated in experiments by taking measurements with both positive and negative $B$. Instead, since the quantum contribution is independent of the angle between the electric and the magnetic fields, in a configuration where they are parallel it is the only term that survives.

Model. Next, we show that monolayer graphene with a (substrate-induced) inversion symmetry-breaking mass as well as TMDs in their trigonal structure support the existence of both the APHE and the nonlinear APHE in the presence of Rashba spin-orbit coupling. To show this, we consider a general microscopic tight-binding model featuring massive 
Dirac cones on the honeycomb lattice,

$$
\begin{aligned}
\mathcal{H}_{c r y}= & -t \sum_{i=1}^{3}\left[\cos \left(\mathbf{k} \cdot \boldsymbol{\delta}_{i}\right) \tau_{x}+\sin \left(\mathbf{k} \cdot \boldsymbol{\delta}_{\boldsymbol{i}}\right) \tau_{y}\right] \otimes \sigma_{0} \\
& +\frac{\Delta}{2} \tau_{z} \otimes \sigma_{0}+\mathcal{H}_{R},
\end{aligned}
$$

where $\sigma$ and $\tau$ refer to the spin and sublattice degrees of freedom, respectively, and $\left\{\boldsymbol{\delta}_{1}, \boldsymbol{\delta}_{2}, \boldsymbol{\delta}_{3}\right\}=$ $\{(0, a / \sqrt{3}),(a / 2,-a / 2 \sqrt{3}),(-a / 2,-a / 2 \sqrt{3})\} \quad$ are the nearest neighbors with $a$ the honeycomb lattice constant. In the Hamiltonian of Eq. (3) the first term containing nerest-neighbor spin-independent hopping respects the $\mathcal{C}_{6 v}$ point-group symmetry of the honeycomb lattice, which is generated by the threefold rotation symmetry $\mathcal{C}_{3 v}=\tau_{x} \otimes e^{i \pi \sigma_{z} / 6}$, the twofold rotation symmetry $\mathcal{C}_{2}=\tau_{x} \otimes e^{i \pi \sigma_{z} / 2}$, and the mirror symmetry $\mathcal{M}_{x}=\tau_{0} \otimes e^{i \pi \sigma_{x} / 2}$. In order to reduce the crystalline symmetry to be trigonal, we have introduced the $\mathcal{C}_{2}$ and inversion-symmetry breaking mass $\propto \Delta$. In graphene, the latter term is naturally realized by placing the graphene flake on lattice-matched substrates, such as hexagonal boron nitride $[49,50]$. Finally, the last term in Eq. (3) is a Rashba-like spin-orbit coupling term that fully breaks the $\mathcal{S U}(2)$ spin symmetry and, therefore, allows for a nonvanishing Berry curvature dipole when an external planar magnetic field is applied. The Rashba term [51] can be written as $\mathcal{H}_{R}=\sqrt{3} \lambda_{R} \sum_{i=1}^{3}\left[\sin \left(\mathbf{k} \cdot \boldsymbol{\delta}_{i}\right) \tau_{x} \otimes\left(\sigma_{y} \boldsymbol{\delta}_{i, 1}-\right.\right.$ $\left.\left.\sigma_{x} \boldsymbol{\delta}_{i, 2}\right)+\cos \left(\mathbf{k} \cdot \boldsymbol{\delta}_{i}\right) \tau_{y} \otimes\left(\sigma_{y} \boldsymbol{\delta}_{i, 1}-\sigma_{x} \boldsymbol{\delta}_{i, 2}\right)\right]$ with the strength of the Rashba coupling $\lambda_{R}$ that in graphene is controlled by the strength of the perpendicular electric field and the local curvature of the graphene sheet [52].

We finally account for the external planar magnetic field introducing the Zeeman coupling term $\mathcal{H}_{Z}=B \tau_{0} \otimes$ $\left(\sigma_{x} \cos \alpha+\sigma_{y} \sin \alpha\right)$ where $\alpha$ is the angle from the zigzag direction of the honeycomb lattice. For $\alpha=2 n \pi / 6$ with $n \in \mathbb{N}$ as the magnetic field preserves one mirror symmetry, thus, allowing only for a Berry curvature dipole. In the absence of spin-orbit interaction, i.e., for $\lambda_{R} \equiv 0$, the Zeeman coupling leads to a closing of the half-filling gap at the critical strength $B_{c} \equiv \Delta / 2$, above which the system becomes a nodal semimetal generated by the crossing of two bands belonging to different spin sectors. A finite value of the Rashba spin-orbit coupling changes the crossings into anticrossings, and, thus, the system has a finite half-filling gap as long as the strength of the applied magnetic field is of the same order of magnitude as the inversion-symmetry breaking mass $\Delta$. For larger values of the applied magnetic-field $B \simeq 2 \Delta$, the half-filling gap closes, but we will neglect this regime in the remainder.

More importantly, a finite value of $\lambda_{R}$ changes the distribution of the Berry curvature allowing for a nonzero Berry curvature dipole. This is explicitly demonstrated in Fig. 2 where we show the local Berry curvature, computed using the method outlined in Ref. [53], both in the absence and in the presence of the Rashba spin-orbit interaction. We find that the effect of the Rashba spin-orbit coupling is twofold. First, it boosts the Berry curvature by reducing the splitting between the two conduction and valence bands. Second, it shifts the dipole distribution away from being centered around the high-symmetry points $K$ and $K^{\prime}$, hence, allowing

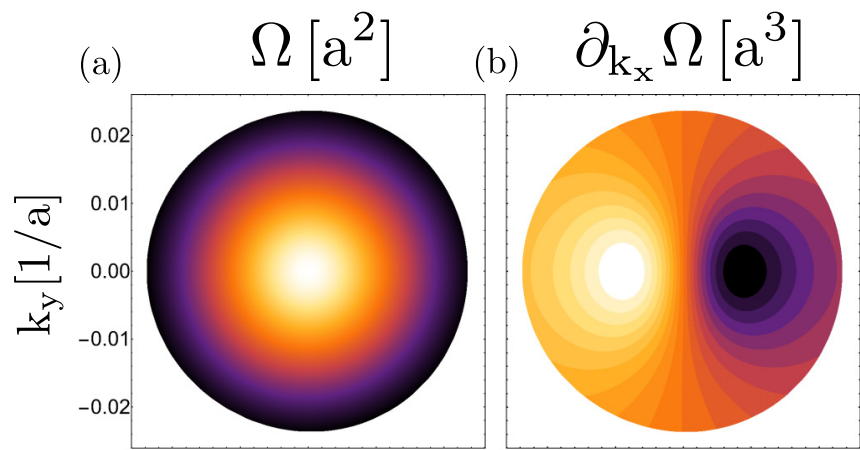

(c) (d)

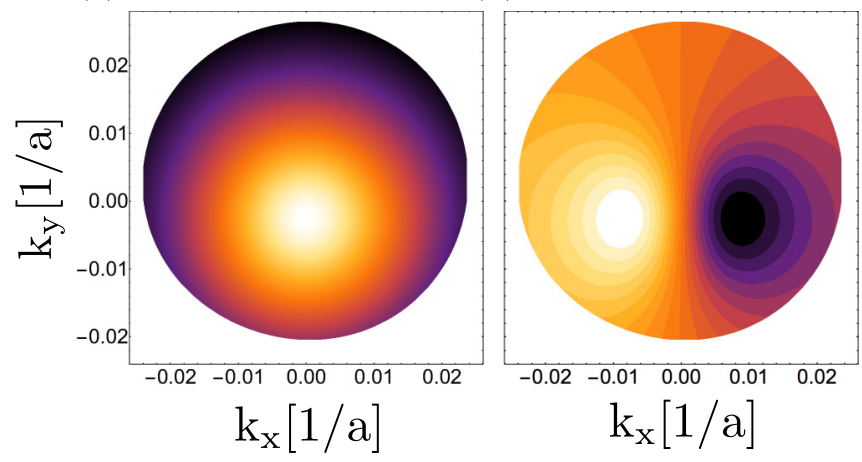

FIG. 2. (a) and (c) Berry curvature $\Omega$ and (b) and (d) dipole density $\partial_{k_{x}} \Omega$ of the conduction bands corresponding to the Hamiltonian of Eq. (3) in the absence (a) and (b) and the presence (c and (d) of Rashba spin-orbit coupling $\left(\lambda_{R} / t=10^{-2}\right)$. The magnetic-field $\left(B / t=10^{-3}\right)$ has been placed along the zigzag direction $\alpha=0$, preserving the mirror symmetry $\mathcal{M}_{x}$. The two valleys at $K$ and $K^{\prime}$ are related by $\mathcal{M}_{x}$ and, hence, contribute identically to the Berry curvature dipole. The inversion breaking mass has been taken to be $\Delta / t=5 \times 10^{-2}$. In plots (b) and (d) light colors correspond to positive values whereas darker colors correspond to negative ones.

for an overall finite dipole. Figure 3(b) shows the behavior of the ensuing Berry curvature dipole as a function of the carrier density for various values of the external planar
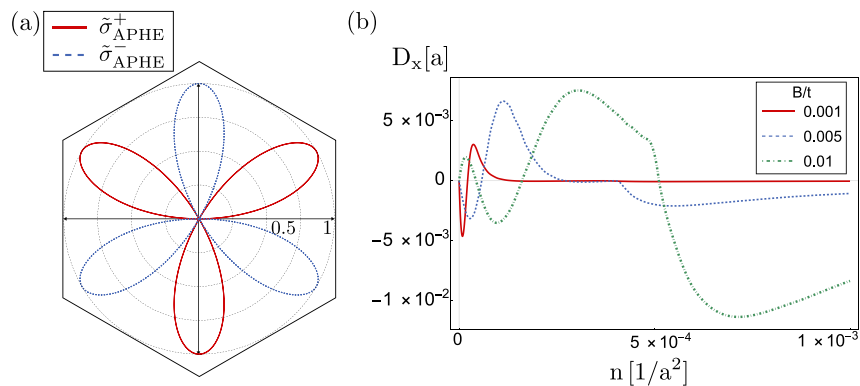

FIG. 3. (a) Polar plot of the anomalous planar Hall conductivity as a function of angle $\alpha$ between the planar magnetic field and the zigzag direction of the honeycomb lattice. The conductivities $\tilde{\sigma}_{\mathrm{APHE}}^{ \pm}$ represent, respectively, the positive and negative part of the full APHE conductivity and are normalized to the maximum at $\alpha=\pi / 2$. The parameters used for the plot are as follows: $\Delta / t=5 \times 10^{-2}$, $B / t=10^{-2}, \lambda_{R} / t=10^{-2}$. (b) Berry curvature dipole $D_{x}$ as a function of the carrier density. Parameters used for the plot are as follows: $\Delta / t=5 \times 10^{-2}, \alpha=0, B / t=10^{-3}, \lambda_{R} / t=10^{-2}$. 
magnetic field. We generally find that increasing the external magnetic-field strength boosts the amplitude of the dipole over a larger range of carrier density. The dipole also displays a characteristic nonmonotonous behavior, similar to the one theoretically predicted and experimentally observed [34,35,37] in the time-reversal nonlinear Hall effect with various sign reversals, which implies that the quantum contribution to the transverse current changes direction. We note that a similar nonmonotonous behavior is also found in the semiclassical symmetric contribution to the nonlinear Hall conductance as shown in the Supplemental Material [45].

Finally, we have computed the linear quantum contribution to the PHE for $\alpha \neq 2 \pi n / 6$. As shown in Fig. 3(a), we find that the integral of the Berry curvature weighed by the equilibrium Fermi distribution contributes to the PHE with an angular dependence that only depends on the relative direction between the magnetic field and the principal crystallographic direction and changes sign under a $\pi$ rotation of the planar magnetic field, in perfect agreement with our general analysis. This dependence is different than the semiclassical contribution $\sigma_{x y}=e^{2} \tau \int_{k} v_{x} v_{y}\left(-\partial f_{0} / \partial \varepsilon_{k}\right)$ [54], which we find to depend exclusively on the angle between the coplanar electric and the magnetic field [45] and follows the usual PHE $\cos \theta \sin \theta$ behavior, thus, vanishing when the applied fields are aligned.
Conclusions. In short, we have shown that twodimensional trigonal crystals with sizable spin-orbit coupling subject to planar magnetic fields display a previously unknown planar Hall effect that contributes to the dissipationless Hall conductance. We dubbed this contribution the anomalous planar Hall effect. This effect is rooted in the geometric properties of the Bloch states encoded in the Berry curvature and appears whenever the planar magnetic field does not leave any residual mirror line. It can be effectively decoupled from the conventional PHE since it survives even when the driving electric field and the planar magnetic field are aligned. Moreover, we have found that in a configuration in which the coplanar fields are aligned and perpendicular to one of the mirror lines of the crystal, transverse Hall currents still exist and appear at second order in the driving electric field. The resulting nonlinear anomalous planar Hall effect has a quantum origin arising from the first moment of the Berry curvature, the Berry curvature dipole. Finally, we propose monolayer graphene on commensurate hexagonal boron nitride substrates as well as transition-metal dichalcognides with trigonal structures as a possible material platform where the (non-)linear APHE can be experimentally observed.

Acknowledgments. C.O. acknowledges support from a VIDI Grant (Project No. 680-47-543) financed by the Netherlands Organization for Scientific Research (NWO).
[1] H. X. Tang, R. K. Kawakami, D. D. Awschalom, and M. L. Roukes, Phys. Rev. Lett. 90, 107201 (2003).

[2] M. Bowen, K.-J. Friedland, J. Herfort, H.-P. Schönherr, and K. H. Ploog, Phys. Rev. B 71, 172401 (2005).

[3] Z. Ge, W. L. Lim, S. Shen, Y. Y. Zhou, X. Liu, J. K. Furdyna, and M. Dobrowolska, Phys. Rev. B 75, 014407 (2007).

[4] N. Wadehra, R. Tomar, R. M. Varma, R. K. Gopal, Y. Singh, S. Dattagupta, and S. Chakraverty, Nat. Commun. 11, 874 (2020).

[5] A. Joshua, J. Ruhman, S. Pecker, E. Altman, and S. Ilani, Proc. Natl. Acad. Sci. USA 110, 9633 (2013).

[6] G. Yin, J.-X. Yu, Y. Liu, R. K. Lake, J. Zang, and K. L. Wang, Phys. Rev. Lett. 122, 106602 (2019).

[7] A. A. Burkov, Phys. Rev. B 96, 041110(R) (2017).

[8] S. Nandy, G. Sharma, A. Taraphder, and S. Tewari, Phys. Rev. Lett. 119, 176804 (2017).

[9] F. C. Chen, X. Luo, J. Yan, Y. Sun, H. Y. Lv, W. J. Lu, C. Y. Xi, P. Tong, Z. G. Sheng, X. B. Zhu, W. H. Song, and Y. P. Sun, Phys. Rev. B 98, 041114(R) (2018).

[10] Z. Wang, Y. Sun, X.-Q. Chen, C. Franchini, G. Xu, H. Weng, X. Dai, and Z. Fang, Phys. Rev. B 85, 195320 (2012).

[11] Z. K. Liu, B. Zhou, Y. Zhang, Z. J. Wang, H. M. Weng, D. Prabhakaran, S.-K. Mo, Z. X. Shen, Z. Fang, X. Dai, Z. Hussain, and Y. L. Chen, Science 343, 864 (2014).

[12] S.-M. Huang, S.-Y. Xu, I. Belopolski, C.-C. Lee, G. Chang, B. Wang, N. Alidoust, G. Bian, M. Neupane, C. Zhang, S. Jia, A. Bansil, H. Lin, and M. Z. Hasan, Nat. Commun. 6, 7373 (2015).

[13] H. Weng, C. Fang, Z. Fang, B. A. Bernevig, and X. Dai, Phys. Rev. X 5, 011029 (2015).

[14] B. Q. Lv, H. M. Weng, B. B. Fu, X. P. Wang, H. Miao, J. Ma, P. Richard, X. C. Huang, L. X. Zhao, G. F. Chen, Z. Fang, X. Dai, T. Qian, and H. Ding, Phys. Rev. X 5, 031013 (2015).

[15] B. Q. Lv, N. Xu, H. M. Weng, J. Z. Ma, P. Richard, X. C. Huang, L. X. Zhao, G. F. Chen, C. E. Matt, F. Bisti, V. N. Strocov, J.
Mesot, Z. Fang, X. Dai, T. Qian, M. Shi, and H. Ding, Nat. Phys. 11, 724 (2015).

[16] S.-Y. Xu, I. Belopolski, N. Alidoust, M. Neupane, G. Bian, C. Zhang, R. Sankar, G. Chang, Z. Yuan, C.-C. Lee, S.-M. Huang, H. Zheng, J. Ma, D. S. Sanchez, B. Wang, A. Bansil, F. Chou, P. P. Shibayev, H. Lin, S. Jia, and M. Z. Hasan, Science 349, 613 (2015)

[17] S.-Y. Xu, N. Alidoust, I. Belopolski, Z. Yuan, G. Bian, T.-R. Chang, H. Zheng, V. N. Strocov, D. S. Sanchez, G. Chang, C. Zhang, D. Mou, Y. Wu, L. Huang, C.-C. Lee, S.-M. Huang, B. Wang, A. Bansil, H.-T. Jeng, T. Neupert, A. Kaminski, H. Lin, S. Jia, and M. Zahid Hasan, Nat. Phys. 11, 748 (2015).

[18] N. P. Armitage, E. J. Mele, and A. Vishwanath, Rev. Mod. Phys. 90, 015001 (2018).

[19] A. Lau and C. Ortix, Phys. Rev. Lett. 122, 186801 (2019).

[20] L. P. He, X. C. Hong, J. K. Dong, J. Pan, Z. Zhang, J. Zhang, and S. Y. Li, Phys. Rev. Lett. 113, 246402 (2014).

[21] T. Liang, Q. Gibson, M. N. Ali, M. Liu, R. J. Cava, and N. P. Ong, Nature Mater. 14, 280 (2015).

[22] C.-L. Zhang, S.-Y. Xu, I. Belopolski, Z. Yuan, Z. Lin, B. Tong, G. Bian, N. Alidoust, C.-C. Lee, S.-M. Huang, T.-R. Chang, G. Chang, C.-H. Hsu, H.-T. Jeng, M. Neupane, D. S. Sanchez, H. Zheng, J. Wang, H. Lin, C. Zhang, H.-Z. Lu, S.-Q. Shen, T. Neupert, M. Zahid Hasan, and S. Jia, Nat. Commun. 7, 10735 (2016).

[23] Q. Li, D. E. Kharzeev, C. Zhang, Y. Huang, I. Pletikosić, A. V. Fedorov, R. D. Zhong, J. A. Schneeloch, G. D. Gu, and T. Valla, Nat. Phys. 12, 550 (2016).

[24] J. Xiong, S. K. Kushwaha, T. Liang, J. W. Krizan, M. Hirschberger, W. Wang, R. J. Cava, and N. P. Ong, Science 350, 413 (2015). 
[25] M. Hirschberger, S. Kushwaha, Z. Wang, Q. Gibson, S. Liang, C. A. Belvin, B. A. Bernevig, R. J. Cava, and N. P. Ong, Nature Mater. 15, 1161 (2016).

[26] M. Z. Hasan and C. L. Kane, Rev. Mod. Phys. 82, 3045 (2010).

[27] A. A. Taskin, H. F. Legg, F. Yang, S. Sasaki, Y. Kanai, K. Matsumoto, A. Rosch, and Y. Ando, Nat. Commun. 8, 1340 (2017).

[28] P. He, S. S.-L. Zhang, D. Zhu, S. Shi, O. G. Heinonen, G. Vignale, and H. Yang, Phys. Rev. Lett. 123, 016801 (2019).

[29] D. Xiao, M.-C. Chang, and Q. Niu, Rev. Mod. Phys. 82, 1959 (2010).

[30] J. E. Moore and J. Orenstein, Phys. Rev. Lett. 105, 026805 (2010).

[31] I. Sodemann and L. Fu, Phys. Rev. Lett. 115, 216806 (2015).

[32] J.-S. You, S. Fang, S.-Y. Xu, E. Kaxiras, and T. Low, Phys. Rev. B 98, 121109(R) (2018).

[33] Y. Zhang, J. van den Brink, C. Felser, and B. Yan, 2D Mater. 5, 044001 (2018).

[34] J. Son, K.-H. Kim, Y. H. Ahn, H.-W. Lee, and J. Lee, Phys. Rev. Lett. 123, 036806 (2019).

[35] S.-Y. Xu, Q. Ma, H. Shen, V. Fatemi, S. Wu, T.-R. Chang, G. Chang, A. M. M. Valdivia, C.-K. Chan, Q. D. Gibson, J. Zhou, Z. Liu, K. Watanabe, T. Taniguchi, H. Lin, R. J. Cava, L. Fu, N. Gedik, and P. Jarillo-Herrero, Nat. Phys. 14, 900 (2018).

[36] Z. Z. Du, C. M. Wang, H.-Z. Lu, and X. C. Xie, Phys. Rev. Lett. 121, 266601 (2018).

[37] Q. Ma, S.-Y. Xu, H. Shen, D. MacNeill, V. Fatemi, T.-R. Chang, A. M. Mier Valdivia, S. Wu, Z. Du, C.-H. Hsu, S. Fang, Q. D. Gibson, K. Watanabe, T. Taniguchi, R. J. Cava, E. Kaxiras, H.Z. Lu, H. Lin, L. Fu, N. Gedik, and P. Jarillo-Herrero, Nature (London) 565, 337 (2019).

[38] J. I. Facio, D. Efremov, K. Koepernik, J.-S. You, I. Sodemann, and J. van den Brink, Phys. Rev. Lett. 121, 246403 (2018).

[39] R. Battilomo, N. Scopigno, and C. Ortix, Phys. Rev. Lett. 123, 196403 (2019).
[40] D. Wawrzik, J.-S. You, J. I. Facio, J. van den Brink, and I. Sodemann, The infinite berry curvature of weyl fermi arcs, arXiv:2010.10537.

[41] A. Kormányos, G. Burkard, M. Gmitra, J. Fabian, V. Zólyomi, N. D. Drummond, and V. Fal'ko, 2D Mater. 2, 022001 (2015).

[42] W. Choi, N. Choudhary, G. H. Han, J. Park, D. Akinwande, and Y. H. Lee, Mater. Today 20, 116 (2017).

[43] S. Manzeli, D. Ovchinnikov, D. Pasquier, O. V. Yazyev, and A. Kis, Nat. Rev. Mater. 2, 17033 (2017).

[44] Y. A. Bychkov and E. I. Rashba, J. Phys. C 17, 6039 (1984).

[45] See Supplemental Material at http://link.aps.org/supplemental/ 10.1103/PhysRevResearch.3.L012006 for a discussion on the planar Hall effects of two- and three-dimensional materials, a derivation of the nonlinear Hall effect in time-reversal symmetric crystals and its connection to Berry curvature dipole, the phase behavior of the model Hamiltonian and the linear and nonlinear semiclassical contributions to the planar Hall effect.

[46] R. Karplus and J. M. Luttinger, Phys. Rev. 95, 1154 (1954).

[47] S. Nandy and I. Sodemann, Phys. Rev. B 100, 195117 (2019).

[48] Y. Gao, S. A. Yang, and Q. Niu, Phys. Rev. Lett. 112, 166601 (2014).

[49] G. Giovannetti, P. A. Khomyakov, G. Brocks, P. J. Kelly, and J. van den Brink, Phys. Rev. B 76, 073103 (2007).

[50] C. R. Woods, L. Britnell, A. Eckmann, R. S. Ma, J. C. Lu, H. M. Guo, X. Lin, G. L. Yu, Y. Cao, R. V. Gorbachev, A. V. Kretinin, J. Park, L. A. Ponomarenko, M. I. Katsnelson, Y. N. Gornostyrev, K. Watanabe, T. Taniguchi, C. Casiraghi, H.-J. Gao, A. K. Geim, and K. S. Novoselov, Nat. Phys. 10, 451 (2014).

[51] C. L. Kane and E. J. Mele, Phys. Rev. Lett. 95, 226801 (2005).

[52] D. Huertas-Hernando, F. Guinea, and A. Brataas, Phys. Rev. B 74, 155426 (2006).

[53] T. Fukui, Y. Hatsugai, and H. Suzuki, J. Phys. Soc. Jpn. 74, 1674 (2005).

[54] G. D. Mahan, Many-Particle Physics, 2nd ed. (Plenum, New York, 1993). 